\title{
LA EDUCACIÓN PERUANA Y EL DESARROLLO SOSTENIBLE
}

\author{
Adriana Luque Ticona'; Susy Laury Barrientos Lazo²; Isaias Rey Pérez Alférez ${ }^{3}$
}

\begin{abstract}
$R E S U M E N$
Los últimos tiempos, altamente demostrativos de los cambios climáticos, obligan a tomar medidas que tiendan a eliminar las posibilidades, anunciada por muchos, de autodestrucción de la humanidad. Uno de los soportes para garantizar la vida, en todas sus formas, es educar. Las organizaciones mundiales como PNUD, asi como muchísimas ONGs, en eventos diferentes y efectuados en casi todas partes del mundo, han incidido sobre la importancia, de carácter vital, del desarrollo de lo que ahora se denomina como Educación Ambiental.
\end{abstract}

\section{$A B S T R A C T$}

The last times, highly demonstrative of the climatic changes, they force to take measures that spread to eliminate the possibilities, announced by many, of the humanity's self-destruction. One of the supports to guarantee the life, in all their forms, is to educate. The world organizations as PNUD, as well as many ONGs, in different events and made in almost all parts of the world, they have impacted about the importance, of vital character, of the development of that that now is denominated as Environmental Education.

\section{IMPORTANCIA DE LAEDUCACIÓN}

La educación es un derecho humano fundamental. La Constitución Política la reconoce, esto en el marco del derecho reconocido en la Declaración Universal de Derechos Humanos y en la Convención sobre los Derechos del Niño. Si bien la Carta Magna lo reconoce y ampara, es más, la declara obligatoria y gratuita en los niveles primario y secundario, en la realidad queda muy lejos de una escolarización y una alfabetización total. Según las informaciones oficiales, manejada el año 2003, en el Perú, son 8600000 los niños y adolescentes en edad escolar; de éstos, sólo 7900000 concurren a las aulas, es decir medio millón no tienen acceso a la educación. Por otro lado, datos del 2002, indican que de la población escolar total matriculada, cerca de medio millón desaprueban (repitencia) y cerca de 200 mil abandona las aulas (deserción). Además de estas cifras también se debe tener en cuenta que millones de niños y niñas asisten a la escuela o colegio en condiciones que hacen que la educación sea de baja calidad tal como infraestructuras deficientes, exceso de alumnos y/o mezcla de diferentes niveles en una misma aula, insuficiencia de profesorado y formación insuficiente, falta de recursos, programas poco adaptados a las necesidades reales, discriminación en el acceso según el sexo o nivel económico, etc.

La mayoría de esta población escolar se encuentra en áreas urbanas donde las condiciones educativas son mejores que en las áreas rurales; el comparativo puede reflejarse en la educación en los paises súper desarrollados fiente a los países menos desarrollados con io que se establece una correlación entre nivel de escolarización y desarrollo. En la Conferencia Mundial sobre Educacion en Jomtien, (1990) y en la Cumbre sobre Desarrollo Social (Copenhague, 1995) se han ido estableciendo metas a nivel mundial, como una educación primaria y gratuita para todos los niños en todo el mundo antes del año 2015, pero no parece que se podrá alcanzar este objetivo fácilmente. Por otro lado la ciencia, que junto con los conocimientos sociales y humanísticos son el principal activo de un país para afrontar su futuro, parece que tampoco podrá solucionar los problemas de desigualdad, de opresión o de subdesarrollo que es el contexto donde viven estos millones de habitantes (Conferencia Mundial sobre la Ciencia de Budapest, 1999).

La educación, criterio que se maneja actualmente, debe favorecer directamente el desarrollo social y económico de una región o un país. También se está de acuerdo que para que esto ocurra, es bésico y previo, el desarrollo de las capacidades personales. Por ello el objetivo fundamental de la educación en general y de la educación escolar en concreto, es proporcionar a los ciudadanos y estudiantes una formación plena que les ayude a estructurar su identidad y a desarrollar sus capacidades para participar en la construcción de la sociedad. En este proceso, el sistema educativo

(1) Licenciada en Educación.

(2) Profesora en Educación.

(3) Antropólogo 
deberia posibilitar que, los alumnos como futuros ciudadanos, reflexionen, construyan y pongan en práctica valores que faciliten la convivencia en sociedades plurales y democráticas, tal como el respeto y la tolerancia, la participación y el diálogo

Por esto, cuando la sociedad en general o un pais concreto se preocupa y se plantea la mejora de su educación, en realidad está confiando en su potencial para generar progreso social y en su potencial transformador en todas las dimensiones: la personal, la política, la cultural y la tecnológica, económica y productiva. $Y$ de forma más inmediata se le está asignando el papel de catalizador para el proceso de adaptación de la sociedad a los acelerados cambios que se producen en cada uno de ellas.

La educación, entonces, debe potenciar las capacidades personales y sociales para hacer frente a las rápidas transformaciones de la tecnologia, de la producción y de la cultura que son fundamentales para el desarrollo de un pais. Pero quizá deberíamos preguntarnos a qué tipo de desarrollo nos referimos. El concepto de desarrollo también está evolucionado y ha pasado de una concepción estrictamente economicista a una concepción más humana, ecologista y sostenible en el futuro, incorporando a este concepto el derecho de las futuras generaciones a vivir en un planeta o un país más equilibrado y más justo (Conferencia de Rio, 1991). Esta interpretación supone la necesidad de un cambio de mentalidad progresiva en todos los ámbitos sociales, en el individual y en el económico, un cambio que fundamentalmente supone entender la educación, formal y no formal, como parte intrínseca e indisociable del desarrollo. Un cambio en el que la educación tiene una función constructiva. $Y$ es por ello que se plantea la necesidad de una Educación para el desarrollo humano, una educación que garantice que el medio será sostenible, es decir una educación para el desarrollo sostenible.

\section{EDUCACIÓN Y CAMBIO}

Muchos estudiosos e intelectuales señalan que estamos viviendo o ingresando en una nueva forma de desarrollo social, la identifican como sociedad de la comunicación y del conocimiento. Igual, se afirma hace tiempo que vivimos en un mundo globalizado o una "aldea" global debido a la era de mundialización de los intercambios en general (materia primas, productos manufacturados, capitales, tecnología, información) y de los procesos productivos (división internacional de las fases de la producción), al desarrollo de las tecnologías y medios de transporte, y al protagonismo de los medios de comunicación. Estas innovaciones hacen que las competencias necesarias para incorporarse a la etapa post modernista deban paralelamente, en el campo educativo, ir cambiando.
En muchos paises las competencias adquiridas, por gran parte de la población, empiezan a ser obsoletas o poco funcionales a corto plazo. Esto, tanto a las capacidades y habilidades personales de los ciudadanos para desenvolverse en la vida cotidiana como a las competencias de la empresa para incorporarse o mantenerse en el mundo productivo o en el mercado. Ya no es suficiente que un pais disponga de mucha mano de obra para ser competitivo, sino que además es necesario que sea cualificada. A cada ciudadano se le empieza a pedir un esfuerzo para que construya su propia cualificación, pero sin que eso suponga ninguna garantia de continuidad en el empleo o de encontrar uno nuevo. $Y$ al mismo tiempo se le impone que olvide conocimientos y experiencias profesionales que siempre habian sido como un seguro personal. Es decir, que en el proceso de globalización y transformación económica, el elemento humano, el trabajador, toma protagonismo por cuanto se le exige más formación, pero al mismo tiempo se siente más vulnerable a los cambios de la organización y necesidades del trabajo y de las empresas. Es decir se genera un sentimiento de temor e inseguridad general ante estas transformaciones. Otra vez se pone de manifiesto que quien marca las necesidades educativas de los ciudadanos en general y de un país en concreto, son las necesidades (económicas, productivas, culturales, etc..) que tiene el sistema para continuar desarrollándose. $Y$ estas necesidades las convierie en factores de competitividad. De esta forma, ante el cambio, la educación se ha de centrar en el desarrollo de capacidades polivalentes y en la formación permanente y reforzar su papel de elemento de integración y promoción social.

¿Qué cambios se deberían introducir para que e ciudadano y trabajador, puedan hacer frente a la nueva situación y a las perspectivas de futuro?. Pergeñemos algunas posibilidades.

En primer lugar se deberia revalorizar el papel de la cultura general, promover y acentuar la identidad, elevar el nivel medio de educación de la población y hacer que la educación básica llegue a toda la población. Esto permitiria que los ciudadanos puedan comprender, crear y adquirir a lo largo de su vida nuevas competencias. Asimismo con esta educación básica se trataría de crear ciudadanos capaces de asimilar innovaciones de tipo tecnológico y cambios económicos, sociales, culturales y de hábitos en general, que continuarán dándose, pero también ciudadanos responsables, democráticos y con capacidad critica.

\section{El potenciar la en la formación básica incidirá en}

- El incremento de la equidad, puesto que permite a los sectores de la población más pobre participar en los procesos de distribución del crecimiento y beneficiarse de nuevas oportunidades. Generaimente 
los momentos de crecimiento económico aumentan las diferencias sociales cuanto mayor es el grado de analfabetismo.

- Fomentar la democracia en tanto que los ciudadanos pueden acceder a más información y más elementos de análisis, pueden conocer mejor sus derechos y pueden participar de forma más autónoma. A niveles mayores de educación mayores controles y demandas políticas y económicas como ampliación del derecho al voto, derechos sindicales, abolición de trabajo infantil, libertad de expresión, etc.

- Construir la estructura sobre la que se desarrollarán las habilidades básicas que con posterioridad permitirán niveles superiores de aprendizaje y de capacitación específica. Las habilidades básicas estarían dirigidas a la expresión oral y escrita, a la resolución de problemas, la capacidad de pensar (capacidad de abstraer, decidir y aprender de la experiencia). Junto a éstas también podriamos considerar como básicas las habilidades sociales e interpersonales ( trabajo en grupo, comunicar ideas, liderar, organizar, etc..), habilidades de comunicación (identificar, adquirir y evaluar información y comunicarla) y de habilidades recursivas (utilización de medios de comunicación y tecnologias más usuales, aprovechar recursos sociales, etc.)

- Posibilitará prevenir las enfermedades y por ende reducir la mortalidad, sobre todo la morialidad infantil.

En ese orden de cosas se debería incrementar la capacitación de tipo laboral. Tradicionalmente las técnicas que se han empleado en la producción siempre han ido cambiando pero de forma progresiva y en base a unos conocimientos básicos, con lo que siempre era fácil que el trabajador pudiese acceder al conocimiento de la nueva técnica (tecnología electromecánica). Pero en la actualidad, la gran dificultad se presenta porque se está modificando la base del conocimiento necesario para ejecutar la técnica (tecnologia electrónica e informática). De esta forma se rompe una cadena de conocimientos y el acceso a la nueva tecnologia ya no es automático, sino que se construyen bajo nuevas estructuras cognitivas de base. Entonces, el trabajador sin formación no puede hacer suyo el saber científico ni la nueva tecnología. Para ello es imprescindible la inversión en educación y en la actualización de la formación profesional. Esto, por un lado, permitiria el incremento del nivel general de conocimientos de la población y, por otro, permitiria acercar la escuela a la empresa. Quizá, y ante la velocidad de las innovaciones tecnológicas, la capacitación básica laboral se debería plantear por grupos de ocupaciones afines o familias con habilidades compartidas.

Finalmente, a pesar de la insistencia en el desarrollo de la educación básica, no se debe olvidar las inversiones en la enseñanza media y superior a pesar de que estos niveles son a los que se les confiere generalmente el papel de transformación y por tanto suelen recibir proporcionalmente más inversiones.

\section{EL MODELO SOSTENIBLE}

Nos preguntábamos, líneas arriba, a qué tipo de desarrollo nos referimos. Es cierto que todos los paises en desarrollo, como es el caso del Perú, tienen como modelo, interiorizado o impuesto el modelo de desarrollo de los países más desarrollados. Pero este modelo está generando una serie de problemas que no son sólo de ámbito interno de cada uno de los paises, sino que tiene implicaciones a nivel mundial y que parece ser que ni la tecnología ni el crecimiento económico puede resolver.

Se dice que el modelo de desarrollo actual es un modelo de desarrollo insostenible desde una perspectiva planetaria. Se sustenta esta afirmación en la generación de una forma de amenaza de destrucción del sistema biológico, que pone en peligro el sistema social y la propia supervivencia humana, que se argumenta por la imposibilidad de que se pueda dar un crecimiento indefinido ( $y$ menos ritmo de los paises más desarroliados) dentro de un planeta limitado y porque la interdependencia entre pobreza y riqueza genera tensiones sociales insostenibles a todas las escalas. De continuar con este modelo, el aumento de la degradación ambiental y la expansión de la pobreza llevaria a un colapso de los sistemas y a una ruptura del orden social tal como ya quedó expresado en la resolución de las Naciones Unidas de 1989.

El problema está en las formas de crecimiento y en los estilos de vida que se fomentan desde el sistema, caracterizado por el neoliberalismo. En consecuencia, todos los países tienen que apostar por un modelo de desarrollo alternativo capaz de asegurar un desarrollo humano y sostenible globalmente. $Y$ decimos, de desarrollo y no de crecimiento, en tanto que desarrollar supone potenciar capacidades que permitan mejorar gradualmente. El compromiso ha de ser tanto de los países en desarrollo como los súper desarrollados pero estos con mucha más responsabilidad moral e implicación económica y tecnológica.. La evaluación de este desarrollo deberá buscar otros indicadores, diferentes a los índices económicos como el PIB o PNB. Un ejemplo, aunque también cuestionado es el Indice de Desarrollo Humano. Esto supone nuevas formas de pensar y de actuar.

\section{DESARROLLO HUMANO SOSTENIBLE}

¿Qué se quiere decir con desarrollo humano sostenible?. Cuando nos referimos a un desarroilo sostenible, estamos planteando la necesidad de que 
este desarrollo ha de cubrir de forma adecuada las necesidades humanas, pero sin transgredir los limites ecológicos de nuestro planeta y que tenga en cuenta las necesidades del presente, sin comprometer las posibilidades de las generaciones futuras para cubrir sus propias necesidades y sin incrementar las desigualdades sociales; en palabras más sencillas, el desarrollo sostenible implica reducir drásticamente, $\mathrm{e}$ inclusive, evitar la contaminación ambiental que, precisamente, es el que genera los más grandes peligros sobre la pervivencia de la vida en la Tierra, que podría ocasionar que el hombre de los países en desarrollo, a futuro, no tengan seguridad de su propia existencia.

Actualmente se manejan dos concepciones alternativas a este dilema. El "oficialista", mantenido por la mayoría de los organismos internacionales de los países desarrollados, que sustentan la creencia que tan solo es posible equilibrar los desniveles del desarrollo entre países ricos y pobres incrementando la producción; al mismo tiempo, confia en que la ciencia y la técnica tienen capacidad para solucionar todos los efectos externos del crecimiento económico (alimentación contaminación, energia, guerras, desigualdades, etc.). Se considera que éstos son efectos no deseables pero inevitables del modelo de libre mercado. El otro enfoque, diriamos "crítico", plantea que en realidad los problemas se derivan del modelo de desarrollo y por tanto el origen estaría en las bases estructurales del mismo modelo: productivismo, competencia económica que se regula de forma natural y la fe en la alianza entre tecnología, ciencia y economía. Por tanto lo que se debería cuestionar es el modelo de desarrollo o mejor dicho de crecimiento. Desde este enfoque critico, humanista y ecológico se considera que el conocimiento científico y técnico es imprescindible en la búsqueda de soluciones, pero se ha de relativizar su capacidad a la vista de los resultados, al tiempo que se piensa que se han de buscar otras formas de regular las relaciones de forma más humana, equitativa y sostenible. En realidad, lo que se considera que se debería cuestionar son los valores que presiden el modelo de desarrollo y las estructuras sociales y económicas de las que se dota. Algunos de estos valores, acuñados desde el neoliberalismo y "aconsejado" por la globalización son el consumismo, utilitarismo, economicismo, individualismo, insolidaridad, competitividad agresiva, etc.

Preguntábamos sobre que entender por desarrollo sostenible $v$ el desarrollo mundial actual. vemos. se caracteriza por las grandes diferencias sociales, económicas, científicas, tecnológicas, etc. Entre el mundo altamente desarrollado con el mundo en desarrollo, caso donde se inscribe nuestra patria. Ello implica un verdadero reto: el definir y concretar un modelo de desarrollo que no suponga grandes regresiones en el nivel desarrollo de los países más avanzados y que permita un desarrollo de los paises menos desarrollados. En ello se trabaja desde el campo económico y político, y a partir de ellos, se podría perfilar algunas características generales de lo que podría ser este modelo de desarrollo humano, global y sostenible: socialmente justo por lo que ha de buscar soluciones a la marginación, a la pobreza y a la distribución desigual; solidario con las futuras generaciones; respetuoso y vigilante de la biodiversidad; pacifista; preservador de la riqueza natural, más colaborador que dominador.

\section{EDUCACIÓN PARA EL DESARROLLO}

Un modelo de desarrollo humano, ecológico y sostenible supone un cambio de mentalidad y una concienciación social de la necesidad de estos cambios. Por ello se deberá intervenir desde la educación formal y no formal a través de una Educación para el Desarrollo. Una educación que es para y en el desarrollo y no sólo para el crecimiento económico, en cuanto que el desarrollo supone un proceso que conduce a la realización y potenciación de capacidades individuales y colectivas.

La Educación para el Desarrollo debería plantear las cuestiones éticas que se relacionan con el funcionamiento de la sociedad y por lo tanto tendrá relación con las problemáticas implicadas entre otras como la paz, la democracia, la multiculturalidad, el consumo, la salud, y el medio ambiente. Se deberia convertir en un ámbito de discusión sobre estas problemáticas relevantes y de formación en actitudes $y$ valores que posibiliten un compromiso personal y colectivo hacia la solución de estas problemáticas. Es decir, en realidad la Educación para el Desarrollo se sitúa en el centro del para qué de la educación. Un para qué que tiene relación con la necesidad de ir hacia la construcción de un modelo de desarrollo diferente, más humano, ecológico y sostenible. Este cambio de paradigma será lento y a contracorriente y mientras tanto tenemos el reto de que el desarrollo de los paises más pobres se ha de aceierar pero sin que eso suponga la competitividad como barbarie, ni un incremento de las desigualdades ni de la exclusión social. El camino del modelo referente utópico hacia el que queremos ir: desarrollo humano y sostenible, es dificil, contradictorio en algunos casos, pero constituye la seguridad para la presencia y vigencia humana sobre el planeta. $Y$ mientras tanto también se deberia crear conciencia social de la necesidad del cambio, desde la escuela en la educación básica, y de forma permanente desde todas las instancias y organizaciones. La Educación para el Desarrollo tiene bastante que decir.

En conclusión, cuando proponemos una educación para el desarrollo, desde los diferentes estamentos y ámbitos de intervención, creemos que estamos 
construyendo las bases para un desarrollo más humano, respetuoso con el medio ambiente y sostenible en el futuro. Estamos proponiendo sentar las bases de la educación para el futuro.

\section{REFERENCIA BIBLIOGRÁFICA}

1. AGENDA 2181992) Conferencia Mundial de las UN o Cumbre de Río.

2. BOTTOMOERE, M. (1998) La ecología del desarrollo humano, Editorial Piados, Barcelona.

3. DELICH, Francisco (1998). Educación, modernidad y democracia: problemas y perspectivas. En Democracia, desarrollo e integración. OEI. Ed.Troquel. Argentina

4. GIMENO SACRISTÁN, J. Y PÉREZ GOMEZ, A. (1993) Comprender y transformar la enseñanza. Editorial Morata, Madrid.
5. GONZALESM. Ma. Del Carmen Principales tendencias y modelos de la educación ambiental en el sistema escolar. www.oei.com

6. INTERMON (1999). Educación ahora: rompamos el círculo de la pobreza. Fundación Intermon/Oxfam Internacional. Barcelona

7. LACUEVA, Aurora (1997) Retos y propuestas para úna didáctica contextualizada. En Educación y Pedagogía IX, Caracas.

8. ONU. (1997) La situación social en el mundo. Departamento de Información Económica y Social y de Análisis de Políticas. ONU. Nueva York. 